\title{
Anhedonia in patients with borderline personality disorder: the efficacy of cognitive-analytic therapy (CAT)
}

\author{
Aris Livanos*1, Aravella Adamopoulou ${ }^{1}$, Konstantinos Katsigiannopoulos ${ }^{1}$, \\ Vasilios Bozikas ${ }^{3}$, Maki Voikli' ${ }^{1}$, John Pandoularis ${ }^{1}$, John Dasoukis ${ }^{1}$, \\ Christina Zagora ${ }^{1}$ and George Garyfallos ${ }^{2}$
}

Address: ${ }^{1}$ Community Mental Health Center of N/W District, Thessaloniki, Greece, ${ }^{22}$ nd Department of Psychiatry, Aristotle University of Thessaloniki, Greece and ${ }^{3} 1$ st Department of Psychiatry, Aristotle University of Thessaloniki, Greece

* Corresponding author

from International Society on Brain and Behaviour: 3rd International Congress on Brain and Behaviour

Thessaloniki, Greece. 28 November - 2 December 2007

Published: 17 April 2008

Annals of General Psychiatry 2008, 7(Suppl I):SI55 doi:I0.II86/I744-859X-7-SI-SI 55

This abstract is available from: http://www.annals-general-psychiatry.com/content/7/SI/SI55

(c) 2008 Livanos et al.; licensee BioMed Central Ltd.

\section{Background}

Anhedonia is recognized as one of the core symptoms of depression but it is also seen in other mental disorders. Cognitive-Analytic Therapy (CAT) is a type of brief psychotherapy which has been proven efficacious for patients with various psychiatric diagnoses. The present study aims to: 1) Investigate the presence of anhedonia in patients with borderline personality disorder (BPD) 2) Evaluate the CAT outcome on anhedonia, depression and anxiety of these patients.

\section{Materials and methods}

The sample of the study consisted of 57 patients, who attended the Mental Health Center of N/W district of Thessaloniki and received a diagnosis of BPD or personality disorder NOS with predominant borderline personality traits according to DSM-IV criteria. These patients completed a 16-sessions of CAT and attended a 2-month follow up. The Beck Depression Inventory (BDI) and the State-Trait Anxiety Inventory (STAI) were used as evaluation instruments both at the intake and at the follow up time. Anhedonia was tested by the relevant question of BDI.

\section{Results}

At the 2-month follow-up, the patients showed a statistically significant improvement on BDI total score, on the anhedonia sub-scale score, as well as on the state and trait scores of the STAI, compared to the intake $(\mathrm{p}<0.001)$. Furthermore, significantly fewer patients were still anhedonic $(18 / 39$ vs $50 / 57, \mathrm{x} 2=37.32, \mathrm{p}<0.001)$ in comparison to pre-therapy evaluation. Finally, patients with BDP only $(\mathrm{N}=21)$ and those with BDP and an additional axis-I diagnosis, except a depressive disorder $(\mathrm{N}=11)$ had a lower score on anhedonia compared to BDP patients with an additional depressive disorder $(\mathrm{N}=25)$. However, the difference showed only a tendency for statistical significance $(\mathrm{p}<0.1)$.

\section{Conclusions}

CAT is an effective psychotherapeutic approach in reducing anhedonia in patients with BDP. The improvement of anhedonia comes into the line with the amelioration of anxiety and depressive symptoms. Anhedonia is a core symptom of a depressive disorder but it is also seen, not infrequently, in patients BDP

\section{References}

I. Collins L, Blanchard J, Biondo K: Behavioral signs of schizophrenia and schizotypy in social anhedonics. Schizophr Res 2005, 78:309-322.

2. Garyfallos G, Adamopoulou A, Voikli M, et al.: Evaluation of Cognitive-Analytic Therapy (CAT) outcome: a 4-8 year follow up. Eur J Psychiatry 2002, 16:197-209. 